\title{
Early Diagnosis and Treatment of Discoid Lupus Erythematosus
}

\author{
Suresh Panjwani, MD, MSc, FRACGP
}

Discoid lupus erythematosus is a chronic dermatological disease that can lead to scarring, hair loss, and hyperpigmentation changes in skin if it is not treated early and promptly. It has a prolonged course and can have a considerable effect on quality of life. Early recognition and treatment improves the prognosis. The diagnosis is usually made by clinical examination. In some cases histopathology may be required to confirm the diagnosis. The histology is that of an inflammatory interface dermatosis. There is insufficient evidence for which treatment is most effective. Because lesions are induced or exacerbated by ultraviolet exposure, photoprotective measures are important. Potent topical steroids and antimalarials are the mainstay of treatment. Some cases of discoid lupus erythematosus can be refractory to standard therapy; in these cases retinoids, thalidomide, and topical tacrolimus offer alternatives, as do immunosuppressives like azathioprine, cyclosporine, mycophenolate mofetil, and methotrexate. (J Am Board Fam Med 2009;22:206-213.)

Lupus erythematosus (LE) is thought to be an autoimmune disease among other connective tissue diseases like scleroderma, rheumatoid arthritis, polymyositis, and mixed connective tissue disease. Within the spectrum of diseases included in LE, at one end is a disease confined mainly to the skin and referred to as discoid lupus erythematosus (DLE) and at the other end is a florid disease with systemic involvement of heart, lungs, brain, kidneys and other organs called systemic lupus erythematosus (SLE). In between the 2 ends of the spectrum are disorders like subacute cutaneous lupus. Subacute cutaneous lupus erythematosus (SCLE) has a rather sudden onset with annular or psoriasiform plaques erupting on the upper trunk, arms, and/or dorsa of hands, usually after exposure to sunlight. ${ }^{1}$ Although at the benign end of the spectrum, $1 \%$ to

This article was externally peer reviewed.

Submitted 13 April 2008; revised 23 June 2008; accepted 1 July 2008.

From Bounces Road Surgery, Forest Primary Care Centre, London, UK.

Funding: none.

Prior presentation: Much of this work was presented as the author's MSc Thesis in Clinical Dermatology; permission for use granted by St. John's Institute of Dermatology, King's College, London.

Conflict of interest: none declared.

Corresponding author: Suresh Panjwani, MD, MSc, FRACGP, 4 Harper Close, Southgate, London N14 4ES, United Kingdom (E-mail: sureshpanjwani18@hotmail.com).
$5 \%$ of patients with discoid lupus may develop $\mathrm{SLE}^{1}$ and $25 \%$ of patients with SLE may develop typical chronic discoid lesions at some time during the course of their illness. ${ }^{2}$

Lupus occurs in all age groups with a mean age varying from 21 years to 50 years ${ }^{3}$ and a prevalence of 17 to 48 in $100,000,{ }^{4}$ with a greater prevalence in Afro-Caribbean people. ${ }^{5}$ Although LE is an autoimmune disease, it is thought to result from an interplay of certain genetic factors, environmental factors like ultraviolet light, and hormonal factors with antibodies.

The diagnosis of discoid lupus is generally made based on clinical features. Histology may be required to confirm the diagnosis; it is that of a lichenoid tissue reaction with changes at the dermo-epidermal junction that include thickening of the basement membrane (best demonstrated by periodic acid-Schiff staining) and vacuolar degeneration of the basal cells along with perivascular and peri-appendageal inflammatory cell infiltration of a variable degree in the reticular dermis. Hyperkeratosis is more evident and follicular plugging may be seen in more mature lesions.

DLE tends to run a less severe course than SLE and has a better prognosis. It is important for family physicians to recognize DLE because it is a potentially scarring disease. Early referral and institution of treatment by dermatologists increases 


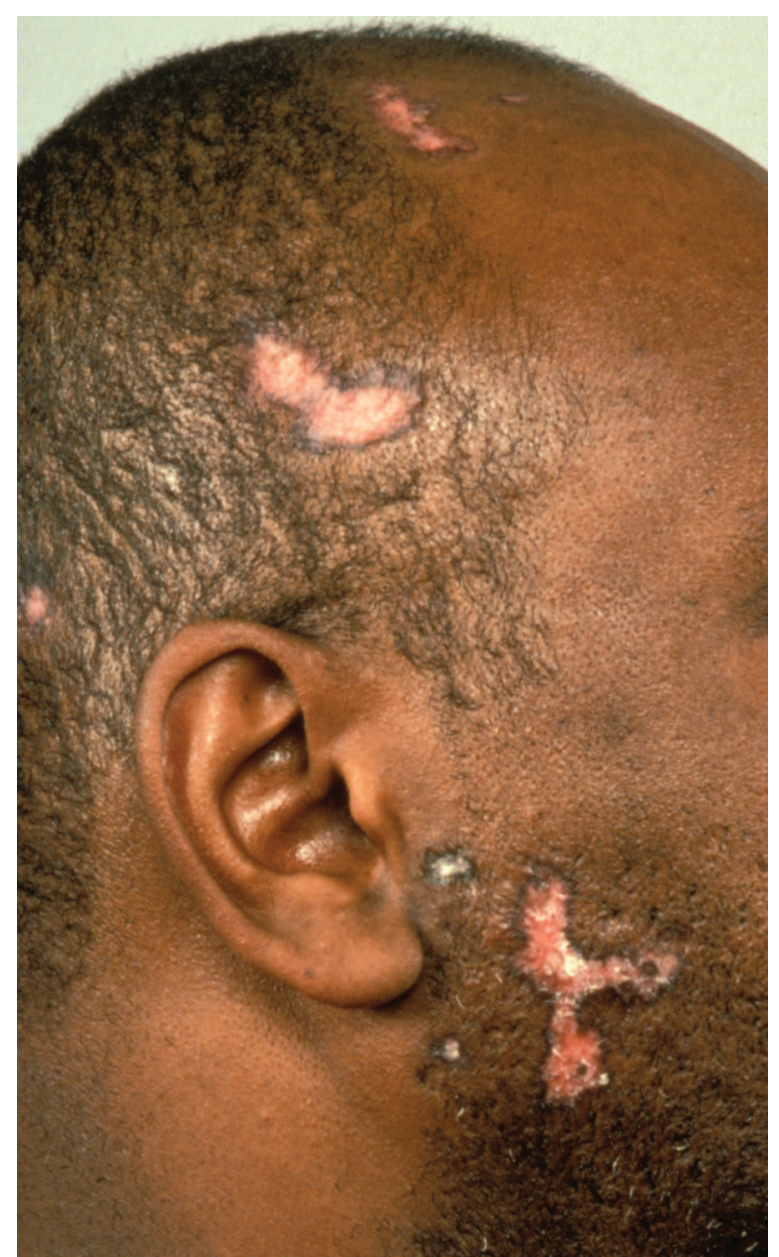

Figure 1. Plaques on beard and scalp in patient with discoid lupus erythematosus.

the hope of minimizing the progression of the disease and consequent socioeconomic impact on the individual.

\section{Clinical Features}

Discoid lupus is by far the most common manifestation of LE. ${ }^{6}$ It commonly presents with erythematous, scaly papules and plaques (Figure 1) occurring on sun-exposed areas, although $50 \%$ of discoid lupus lesions are found on areas of hair-bearing scalp that are presumably protected from the $\operatorname{sun}^{6}$ (Figures 2 and 3). In the localized variety of discoid lupus the lesions tend to be confined to the head and neck and in the generalized variety they occur both above and below the neck. Patients with generalized discoid have significantly greater chances of having laboratory abnormalities and of progressing to systemic LE. Most people with DLE do not

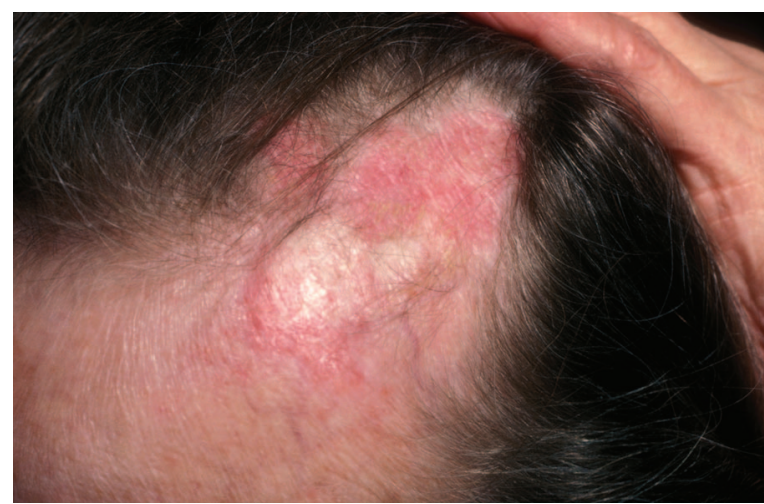

Figure 2. Critical alopecia on scalp caused by discoid lupus erythematosus.

have any systemic or serologic abnormality although antinuclear antibodies may be present.

Discoid lupus occurs at all ages and among all ethnic groups; it occurs more frequently in women than in men, but the predilection among women is not as marked as in systemic lupus. Discoid lupus starts as an erythematous papule or plaque, usually on the head or neck, with an adherent scale. The lesion tends to spread centrifugally and as it progresses there is follicular plugging and pigmentary changes, generally hyperpigmentation at the periphery, and hypopigmentation with atrophy, scarring, and telengiectasia at the center of the lesion (Figure 4).

Involvement of the scalp commonly produces a scarring alopecia, ${ }^{6}$ but there has been an increase in incidence of alopecia areata among patients with LE. ${ }^{7}$ Scarring alopecia was present in $34 \%$ of 89 patients with DLE and was associated with a prolonged disease course. More than half of those patients had scalp disease at the onset. ${ }^{7}$ There are no reliable predictors of scalp involvement. Histologically there is a perifollicular lymphocytic inflammation maximal around mid-follicle. The midfollicle is in fact a very important structure becuase it contains the bulge that contains the follicular stem cells.

\section{Treatment of Discoid Lupus Erythematosus}

DLE is a scarring autoimmune disease that can linger on for a prolonged period, not surprisingly, the psychological impact is considerable. ${ }^{4}$ Consequently there is a need for treatment, often prolonged, that incurs considerable expenditure for health facilities. 


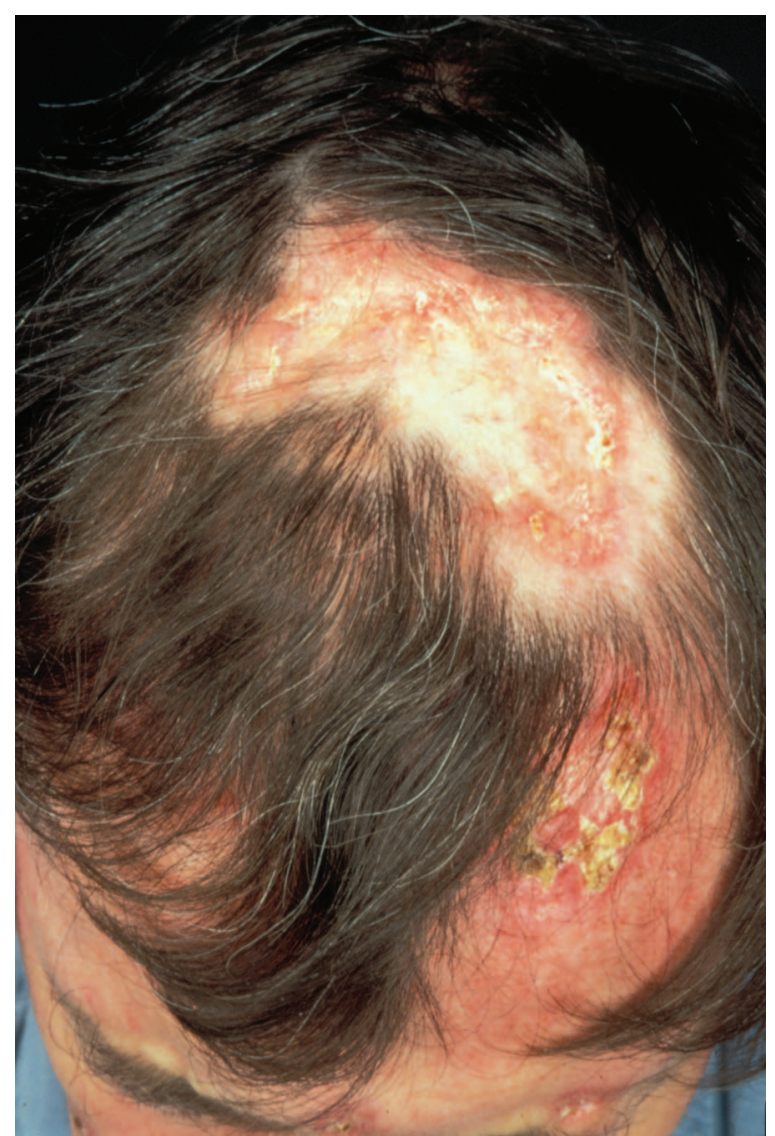

Figure 3. Extensive loss of scalp hair in a patient with discoid lupus.

Early effective treatment may lead to total clearing of skin lesions, but failure of treatment results in permanent scarring; the depressed scars, hair loss, and pigmentary changes are often extremely disfiguring, particularly in darker-skinned people. ${ }^{8}$ According to a 2004 systematic review of treatment of discoid lupus by Jessop et $\mathrm{al}^{8}$ only 30 trials were identified through a search of the Cochrane Clinical Trials Register (December 1999); Medline (January 1966 to December 1999); Embase (January 1980 to January 2000); and Index Medicus (1956 to 1966). ${ }^{8}$ Only 4 of these were controlled trials and only 2 of the latter were randomized (A, level 2). Accordingly, more evidence is needed to guide clinicians to the best treatment options for DLE, particularly for the severe type.

The treatment of DLE would in most instances be initiated at a dermatology department, but before instituting treatment for discoid lupus patients should be assessed for systemic involvement. This should include a full history and physical examination, full blood count, erythrocyte sedimentation

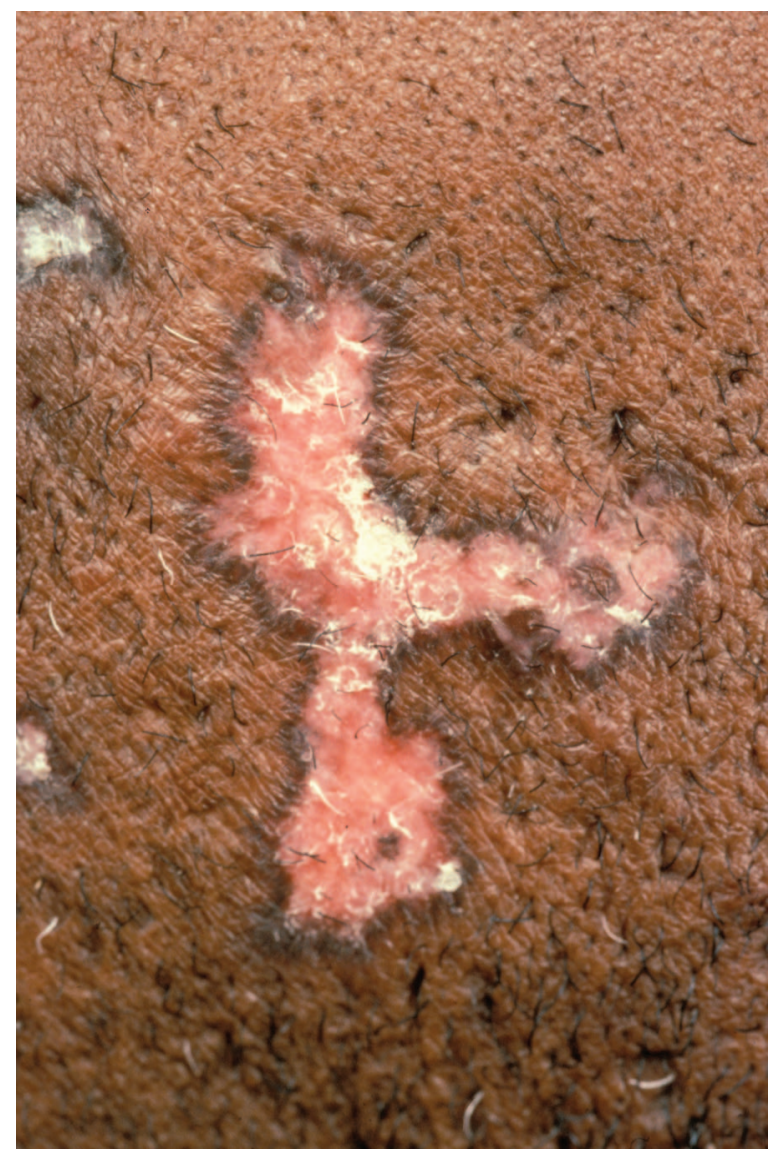

Figure 4. Close-up view of plaque in a patient with discoid lupus.

rate, midstream urine, and antinuclear antibody. ${ }^{9}$ If SLE is suspected, anti-double stranded DNA, extractable nuclear antigen, $\mathrm{C} 3 / \mathrm{C} 4$, and renal review should also be included. ${ }^{?}$

\section{General Measures}

Because cutaneous lesions of lupus are known to be induced or exacerbated by exposure to ultraviolet light, a logical approach in the management of discoid lupus must include sun avoidance and the liberal application of sunscreens. Patients should be educated about the use of sunscreens and protective clothing and behavior modification to avoid sun exposure, particularly between $10 \mathrm{AM}$ and $4 \mathrm{PM}$. They should also be aware of water, snow, and sand surfaces, from which ultraviolet light may be reflected, and where harm can occur from reflected ultraviolet light.

They should be instructed to use sunscreens daily and apply liberally; they should reapply them if there has been prolonged sun exposure or when 
they are wet. ${ }^{10}$ Protection against both ultraviolet $\mathrm{A}$ and ultraviolet B is desirable because lupus is aggravated by both. ${ }^{11,12}$ With disfigurement and alopecia, patients may benefit from advice on camouflage and the wearing of a wig.

\section{Topical Corticosteroids}

Topical steroids are the mainstay of treatment of DLE. Patients usually start with a potent topical steroid applied twice a day, then switch to a lowerpotency steroid as soon as possible. The minimal use of steroids reduces the recognized side effects like atrophy, telengiaectasiae, striae, and purpura.

\section{Intralesional Steroids}

Intralesional steroids are particularly useful to treat chronic lesions, hyperkeratotic lesions, and those that do not respond adequately to topical steroids. Lesions at particular sites, eg, the scalp, may also benefit. Recognized side effects of intralesional steroids include cutaneous atrophy and dyspigmentation, which are not significant risks in experienced hands. ${ }^{13}$ Oral steroids may be required for the control of systemic lupus but are not generally beneficial in DLE. For patients with progressive or disseminated disease or in those with localized disease that does not respond to topical measures, the addition of systemic agents should be considered.

\section{Antimalarials}

Treatment with antimalarial drugs constitutes firstline systemic therapy for DLE. Therapy with antimalarials, either used singly or in combination, is usually effective. ${ }^{14}$ The 3 commonly used preparations include chloroquine, hydroxychloroquin, and mepacrine. Mepacrine is not freely commercially available in the United States but is freely available in other countries like the United Kingdom.

It is customary to start hydroxychloroquine at a dose of $200 \mathrm{mg}$ per day for an adult and, if there are no untoward gastrointestinal or other side effects, to increase the dose to twice a day. No more than $6.5 \mathrm{mg} / \mathrm{kg} /$ day should be administered. It is important to emphasize to the patient that it may take between 4 to 8 weeks for any clinical improvement. In some patients who do not respond to hydroxychloroquine, chloroquine may be more effective. Some patients do not respond well to monotherapy with either hydroxychloroquine or chloroquine, and in such cases the addition of mepacrine may be of benefit. ${ }^{15}$
In general, hydroxychloroquine and mepacrine are safe, well-tolerated drugs and adverse effects are relatively few, the most widely recognized being retinal toxicity. ${ }^{16}$ Chloroquine causes macular pigmentation that progresses to a typical bull's eye lesion and then to widespread retinal pigment epithelial atrophy resembling retinitis pigmentosa. ${ }^{16}$ This is dose related and can largely be avoided. The side effect spectrum between chloroquine and hydroxychloroquine is different, with ocular toxicity being mainly, although perhaps not exclusively, seen after chloroquine use. To prevent overdosing, doses should be calculated not on the actual weight of the patient but on ideal (lean) body weight; ${ }^{17}$ this substantially reduces the risk of retinal toxicity.

Other adverse effects of antimalarials include gastrointestinal symptoms, eg, nausea and vomiting, and cutaneous side effects including pruritus, lichenoid drug reactions, annular erythema, hyperpigmentation, and hematological disturbances like leukopenia and thrombocytopenia. ${ }^{18}$ Hemolysis is reported in individuals who are deficient in the enzyme glucose-6-phosphate-dehydrogenase. Hydroxychloroquine has, on rare occasions, caused toxic psychosis when used for the treatment of discoid lupus. ${ }^{19}$ Prolonged mepacrine therapy may produce a yellow discoloration of the skin and urine. Hepatitis and aplastic anemia have also been reported.

Potentially more toxic therapeutic medication needs to be used in the management of many cases of DLE; however, topical tacrolimus ointment has been found recently to be useful in the management of DLE (see below). The different forms of therapeutic agents used in the management of DLE are highlighted in Table 1.

Thalidomide may provide one of the most useful therapeutic alternatives for chronic refractory DLE, although its distribution is limited to a few countries because of the risk of teratogenicity and polyneuropathy. ${ }^{20}$ However, in a retrospective study of 18 patients with chronic DLE, Brocard et $\mathrm{al}^{21}$ found low-dose thalidomide treatment was efficacious with good tolerance, with the most frequent side effect being usually mild asthenia.

\section{Other Drugs Used for the Treatment of DLE} Methotrexate

In 1995, Bottomley and Goodfield ${ }^{22}$ found that methotrexate may be of help to patients with DLE resistant to conventional treatment; short-term 


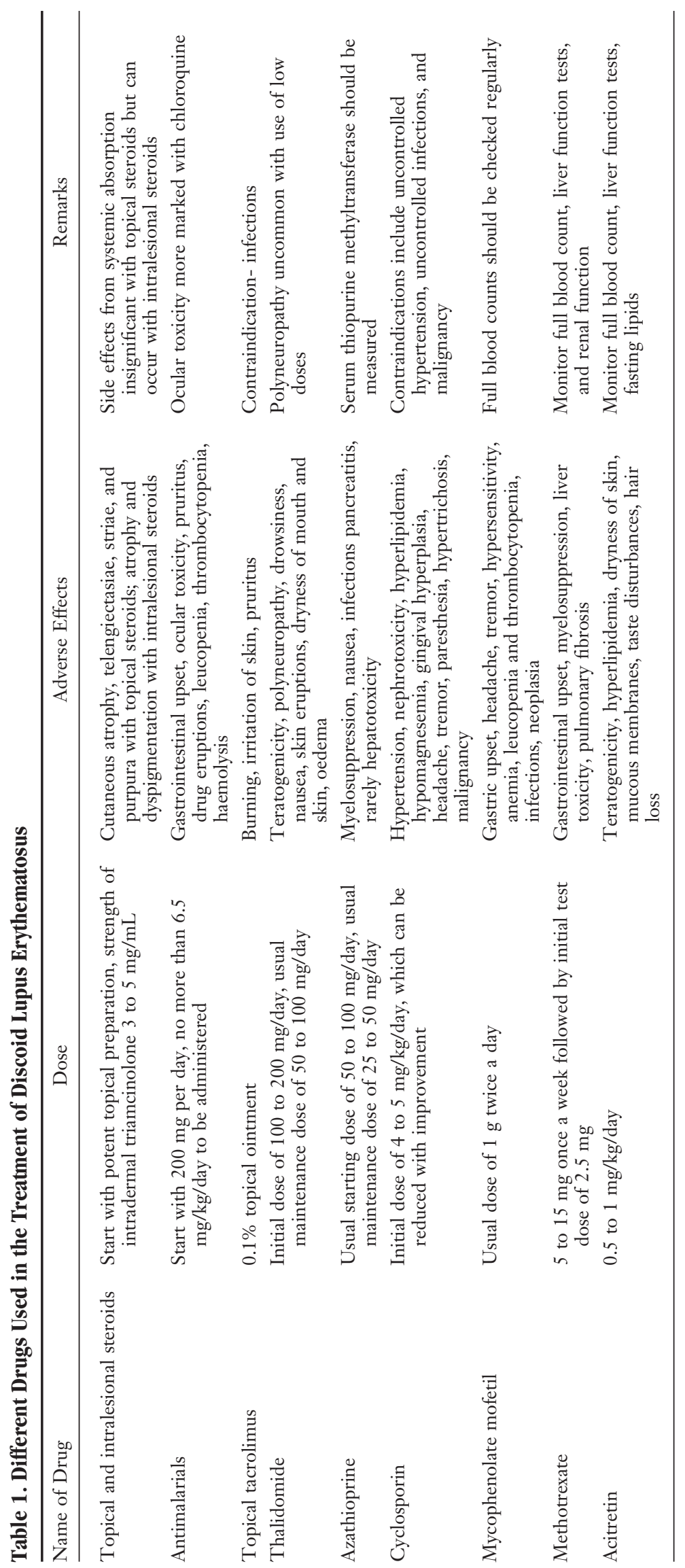


treatment is unlikely to be complicated by any significant side effects. ${ }^{22}$ Full blood count and liver function along with renal function need to be checked before commencing treatment with methotrexate and regularly thereafter because it can cause myelosuppresion and hepatic and renal impairment.

\section{Cyclosporin A}

This is a potent immunosuppressant because of its immunomodulating effect on helper T-cell function, inhibiting lymphocyte activation and proliferation. Because DLE is an inflammatory dermatosis with T-cell infilterate it should not be surprising if cyclosporine is effective in the management of the condition. In 1994 Yell and Burge ${ }^{23}$ tried cyclosporine in 2 patients with severe DLE and concluded that it was effective at a dose of 4 to 5 $\mathrm{mg} / \mathrm{kg} /$ day, but others have not confirmed this finding. Blood pressure and kidney function need to be monitored, and hypertension is a common side effect. It can also cause gingival hyperplasia and hirsutism. Lipid disturbances can also occur and therefore serum cholesterol and triglycerides have to be monitored.

\section{Tacrolimus}

Tacrolimus is a macrolide derived from the fungus Streptomyces tsukubaensis and has been used in recent years to treat a number of inflammatory and autoimmune conditions. When used as an ointment it acts as a local immunosuppressive agent. Walker et $\mathrm{al}^{24}$ reported 2 patients with severe recalcitrant chronic discoid lupus that had not responded to potent topical steroids or antimalarials but dramatically responded to topical tacrolimus ointment in one case and a combination of clobetasol ointment and tacrolimus in the other. ${ }^{24}$ Recently, Tzung et $\mathrm{al}^{25}$ conducted a randomized double-blind study in which 20 patients were enrolled but only 11 women and 7 men (13 with malar rash of SLE, 4 with DLE, and 1 with SCLE) completed the study. All patients had facial cutaneous LE and were instructed to apply $0.1 \%$ tacrolimus ointment twice daily to the affected areas on one side of the face and $0.05 \%$ clobetasol propionate ointment on the other side; this was randomly assigned for each patient. The severity of lesions was assessed at each visit (weeks 0-4 and posttreatment week 4) using a 7-point rating scale. They found tacrolimus was as efficient as clobetasol in treating cutaneous LE (B, level 2). ${ }^{25}$

\section{Mycophenolate mofetil}

This is an immunosuppressive agent that has been added relatively recently to the other drugs in this group and has been used increasingly in recent years for the treatment of various dermatoses that are inflammatory or autoimmune in origin. Mycophenolate is an ester prodrug of mycophenolic acid, initially isolated from Penicillium species. ${ }^{26}$ Goyal and Nousari ${ }^{27}$ described 2 cases of refractory discoid lupus involving the palms and soles that responded satisfactorily to mycophenolate mofetil.

\section{Azathioprine}

Azathioprine, a potentially toxic drug, has been used in refractory cases of discoid lupus, with particular success among those with the involvement of the palms of the hands and the soles of the feet. ${ }^{28}$ It is a synthetic derivative of 6-mercaptopurine and is an immunosuppressive drug. There are wide differences in the activity of the enzyme thiopurine methyltransferase in different individuals, which can be measured by a blood test. The chances of myelosuppression in a patient with very low levels of thiopurine methyltransferase are significantly greater than in others.

Other forms of treatment have recently been found to be useful in the treatment of DLE. Gul et $\mathrm{al}^{29}$ described a case of generalized DLE successfully treated with $5 \%$ Imiquimod cream applied to lesions once a day 3 times a week. After 20 applications all of the lesions regressed significantly. Usmani and Goodfield ${ }^{30}$ reported good to excellent responses in 12 out of 13 patients with DLE who were treated with efalizumab, a monoclonal antibody directed against CD 11a (discoid lupus is known to be predominantly t-cell mediated). Finally, Koch et al ${ }^{31}$ suggest cryotherapy as a treatment option in cases of DLE lesions that are resistant to local or systemic recommended therapy. The standard therapies for the management of cutaneous lupus, including sunscreens, protective clothing, and behavioral alteration, and topical steroids with or without an antimalarial agent are often not used appropriately and can result in a situation in which the patient has a refractory disease. $^{32}$

\section{Conclusion}

DLE is a chronic scarring and potentially disfiguring disease seen in all parts of the world and among 
all ethnic groups. It is an important cause of irreversible hair loss and is associated with considerable morbidity. It is extremely important for family physicians to diagnose this relatively uncommon condition early because early effective treatment is important to promote the resolution of established lesions and to prevent scarring. There are several forms of treatment that are effective to a lesser or greater degree than others. There are too few properly conducted randomized trials to enable an informed choice by clinicians. Clinicians at the present time are, therefore, likely to choose their preferred treatment based on their own experience. There is a need for further large randomized, controlled, and possibly multinational trials to be conducted that compare the effectiveness and safety of one form of treatment compared with another.

\section{References}

1. Fitzpatrick TB, Johnson RA, Klaus W, Suurmond D. In colour atlas and synopsis of clinical dermatology, 4th ed. New York (NY): McGraw-Hill Companies; 2001:368-9.

2. Lahita RG. In systemic lupus erythematosus, 2 nd ed. New York (NY): Churchill Livingstone; 1987:620.

3. Tebbe B, Orfanos CE. [Lupus erythematosus of the skin. An analysis of 97 patients.] Z Hautkr 1987;62: 1563-72, 1577-8, 1583-4.

4. Tebbe B, Orfanos CE. Epidemiology and socioeconomic impact of skin disease in lupus erythematosus. Lupus 1997;6:96-104.

5. Hochberg MC, Boyd RE, Ahearn JM, et al. Systemic lupus erythematosus: a review of clinico-laboratory features and immunogenetic markers in 150 patients with emphasis on demographic subsets. Medicine 1985;64:285-95.

6. Hymes SR, Jordon RE. Chronic cutaneous lupus erythematosus. Med Clin N Am 1989;73:1055-71.

7. Werth VP, White WL, Sanchez MR, Franks AG. Incidence of alopecia areata in lupus erythematosus. Arch Dermatol 1992;128:368-71.

8. Jessop S, Whitelaw D, Jordaan F. Drugs for discoid lupus erythematosus. Cochrane Database Syst Rev 2001;(1):CD002954.

9. Donnelly AM, Halbert AR, Rohr JB. Discoid lupus erythematosus. Australas J Dermatol 1995;36:3-10; quiz 11-2.

10. Ting WW, Sontheimer RD. Local therapy for cutaneous and systemic lupus erythematosus: practical and theoretical considerations. Lupus 2001;10:17184.

11. Sanders CJ, Van Weelden H, Kazzaz GA, Sigurdsson V, Toonstra J, Bruijnzeel-Koomen CA. Photosensitivity in patients with lupus erythematosus: a clinical and photobiological study of 100 patients using a prolonged phototest protocol. Br J Dermatol 2003;149:131-7.

12. Lehmann P, Holzle E, Kind P, Goerz G, Plewig G. Experimental reproduction of skin lesions in lupus erythematosus by UVA and UVB radiation. J Am Acad Dermatol 1990;22:181-7.

13. Callen JP. Cutaneous lupus erythematosus: a personal approach to management. Australas J Dermatol 2006;47:13-27.

14. Callen JP. Treatment of cutaneous lesions in patients with lupus erythematosus. Dermatol Clin 1994;12:201-6.

15. von Schmiedeberg S, Rönnau AC, Schuppe HC, Specker C, Ruzicka T, Lehmann P. [Combination of antimalarial drugs mepacrine and chloroquine in therapy refractory cutaneous lupus erythematosus.] Hautarzt 2000;51:82-5.

16. Cruz DD. Antimalarial therapy: a panacea for mild lupus. Lupus 2001;10:148-51.

17. Mackenzie AH. Dose refinements in the long term therapy of rheumatoid arthritis with antimalarials. Am J Med 1983;75:40-5.

18. Lo JS, Berg RE, Tomecki K. Treatment of discoid lupus erythematosus. Int J Dermatol 1989;28:497505.

19. Ward Q, Walter-Ryan WG, Shehi GM. Toxic psychosis: a complication of antimalarial therapy. J Am Acad Dermatol 1985;12:863-5.

20. Fabbri P, Cardinali C, Giomi B, Caproni M. Cutaneous lupus erythematosus: diagnosis and management. Am J Clin Dermatol 2003;4:449-65.

21. Brocard A, Barbarot S, Milpied B, Stalder JF. Thalidomide in the treatment of chronic discoid lupus erythematosus. Ann Dermatol Venereol 2005;132 (11 Pt 1):853-6.

22. Bottomley WW, Goodfield MJ. Methotrexate for the treatment of discoid lupus erythematosus. Br J Dermatol 1995;133:655-6.

23. Yell JA, Burge SM. Cyclosporin and discoid lupus erythematosus. Br J Dermatol 1994;131:132-48.

24. Walker SL, Kirby B, Chalmers RJ. The effect of topical tacrolimus on severe recalcitrant chronic discoid lupus erythematosus. Br J Dermatol 2002;147: 405-6.

25. Tzung TY, Liu YS, Chang HW. Tacrolimus vs. clobetasol propionate in the treatment of facial cutaneous lupus erythematosus: a randomized, doubleblind, bilateral comparison study. $\mathrm{Br} \mathrm{J}$ Dermatol 2007;156:191-2.

26. Nousari HC, Sragovich A, Kimyai-Asadi A, Orlinsky D, Anhalt GJ. Mycophenolate mofetil in autoimmune and inflammatory skin disorders. J Am Acad Dermatol 1999;40(2 Pt 1):265-7.

27. Goyal S, Nousari HC. Treatment of resistant discoid lupus erythematosus of palms and soles with mycophenolate mofetil. J Am Acad Dermatol 2001;45: $142-4$. 
28. Abu Shakra M, Shoenfield Y. Azathioprine therapy for patients with systemic lupus erythematosus. Lupus 2001;10:152-3.

29. Gül U, Gönül M, Cakmak SK, Kiliç A, Demiriz M. A case of generalized discoid lupus erythematosus: successful treatment with imiquimod cream $5 \%$. Adv Ther 2006;23:787-92

30. Usmani N, Goodfield M. Efalizumab in the treat- ment of discoid lupus erythematosus. Arch Dermatol 2007;143:873-7.

31. Koch M, Horwath-Winter J, Aberer E, Salmhofer W, Klein A. [Cryotherapy in discoid lupus erythematosus (DLE).] Ophthalmologe 2008;105:381-3.

32. Callen JP. Management of refractory skin disease in patients with lupus erythematosus. Best Pract Res Clin Rheumatol 2005;19:767-84. 